\title{
Reply to "Discussion: The kinetics of blood lactate in boys during and following a single and repeated all-out sprints of cycling are different than in men - Do children indeed release and remove lactate faster than adults?"
}

\author{
Florian Azad Engel, Sascha Härtel, Billy Sperlich, and Hans-Christer Holmberg
}

\section{Introduction}

It was with interest that we read the comments by Dotan and Falk (2015) concerning our recent publication (Engel et al. 2015) and we appreciate their contributions to this complex aspect of pediatric physiology. These researchers pose important questions regarding the biexponential model we employed to interpret our data and argue for an alternative model on the basis of their own previous findings (Dotan et al. 2003). We would like to respond to their major comments as follows:

First, Falk and Dotan question the validity of the biexponential model we applied (Engel et al. 2015) since this model was designed by Dost to evaluate pharmokinetics (Dost 1968), which typically involve hours or days, whereas lactate responses occur in minutes and seconds.

To begin with, this biexponential model has been applied successfully in various settings (Rimaud et al. 2010; Messonnier et al. 2006; Beneke et al. 2005) and, more importantly, has been validated in adult men. When Freund and Zouloumian (1981) assayed lactate in arterial and venous blood at 10- or 30-s intervals before, during, and after several types of exercise, the curves they calculated for the arterial lactate concentration during recovery revealed a biexponential process consisting of a rapidly increasing and slowly decreasing component. Our curve fits the experimental data well $(r=0.9)$, indicating the validity of our model. This good fit with the experimental data for the boys (a required condition), together with the validation of the model in men (Freund and Zouloumian 1981) were the crucial factors that motivated us to apply this model to lactate kinetics in boys. To the best of our knowledge, no other model has either proven to provide a closer fit or been evaluated invasively in children, which in our opinion is required for validation.

Second, in their own study, Dotan et al. (2003) demonstrated that when postexercise lactate concentrations were similar, lactate was eliminated from the blood of boys and men at the same rate. In their comments on our study (Dotan and Falk 2015), they state that children do not possess any "secret weaponry" when it comes to metabolizing lactate (a statement we fully agree with) that could explain their more rapid recovery, in general, and from high-intensity exercise, in particular.
On the basis of their previous findings (Dotan et al. 2003), these investigators discuss the half-life of lactate in children versus adults. To our way of thinking, it is problematic to compare their methodology with our approach, since the exercise performed by their subjects was totally different (Engel et al. 2015). While we analyzed lactate kinetics in boys and men following exercise of the same duration and high intensity, Dotan and colleagues had their men and boys perform sprints of different durations to achieve the same peak lactate concentrations, following which the half-life of blood lactate recovery was the same in both groups.

In this context we have recalculated the half-life of lactate, as described elsewhere (Kappenstein et al. 2015), following a single and four 30-s sprints by boys and men employing both our biexponential model and the model proposed by Dotan and co-workers (2003) (integrating the half-life calculation of blood lactate kinetic). First, we observed significantly shorter half-lives with both types of exercise for the boys than men (Table 1) and, interestingly, the peak lactate values for the men following the single sprint were quite similar to those for the boys following the four 30-s sprints (men: $11.5 \pm 2.1$ vs. boys: $12.2 \pm 3.6 \mathrm{mmol} / \mathrm{L}$ ). When we then apply an approach similar to that of Dotan et al. (2003) we find that the half-life for the boys was $2 \mathrm{~min}$ shorter than for the men. We are well aware that the neurological, peripheral, and central stress induced by a single sprint by the men and four sprints by the boys may differ, but this may also be the case in connection with the sprints of different durations employed by Dotan and colleagues. Nonetheless, the present calculations show that the half-life of lactate in boys may very well be shorter than in men.

The calculations described here agree with those by Kappenstein et al. (2015), who found that following repeated high-intensity sprints, the half-life of lactate is shorter in children than adults, in good accord with our own findings (Engel et al. 2015). In both studies with either similar or considerably lower peak lactate concentrations the boys appeared to eliminate lactate more rapidly. Thus, this shorter half-life supports the more rapid postexercise elimination of lactate indicated by our biexponential model (Engel et al. 2015).

To summarize: The suggestion by Dotan and Falk (2015) that to account for their different body sizes a 3-, rather than a 2-component model be employed to compare lactate kinetics in

Received 5 March 2015. Accepted 9 March 2015.

F.A. Engel. Research Centre for School Sports and the Physical Education of Children and Adolescents, Karlsruhe Institute of Technology, Karlsruhe, Germany; Integrative and Experimental Training Science, Department of Sport Science, University of Würzburg, Würzburg, Germany.

S. Härtel. Institute of Sports and Sports Science, Karlsruhe Institute of Technology, Karlsruhe, Germany.

B. Sperlich. Integrative and Experimental Training Science, Department of Sport Science, University of Würzburg, Würzburg, Germany.

H.-C. Holmberg. Department of Health Sciences, Swedish Winter Sports Research Centre, Mid Sweden University, Östersund, Sweden.

Corresponding author: Florian Engel (e-mail: engel@foss-karlsruhe.de). 
Table 1. The peak concentration, half-life and velocity constants $\gamma_{1}$ and $\gamma_{2}$ of blood lactate in boys and men following a single and four 30-s sprints.

\begin{tabular}{lcclll}
\hline & \multicolumn{2}{c}{ Single sprint } & & \multicolumn{2}{l}{ Four sprints } \\
\cline { 2 - 3 } \cline { 5 - 6 } \cline { 5 - 6 } & Boys & Men & & Boys & Men \\
\hline $\begin{array}{l}\text { Peak lactate concentration } \\
(\text { mmol/L) }\end{array}$ & $8.7 \pm 1.8^{*}$ & $11.5 \pm 2.1$ & & $12.2 \pm 3.6^{*}$ & $16.1 \pm 3.3$ \\
$\begin{array}{c}\text { Half-life time of blood lactate } \\
(\text { min:s) }\end{array}$ & $17: 20 \pm 4: 00^{*}$ & $22: 00 \pm 2: 15$ & & $19: 56 \pm 4: 55^{*}$ & $26: 00 \pm 3: 5$ \\
$\gamma_{1}\left(\mathrm{~min}^{-1}\right)$ & $1.45 \pm 0.65$ & $0.584 \pm 0.24^{*}$ & & $0.234 \pm 0.05$ & $0.222 \pm 0.05$ \\
$\gamma_{2}\left(\mathrm{~min}^{-1}\right)$ & $0.059 \pm 0.023$ & $0.043 \pm 0.013^{*}$ & & $0.049 \pm 0.016$ & $0.036 \pm 0.014^{*}$ \\
\hline
\end{tabular}

*Significant difference between the men and boys $(P<0.05)$ as determined by the $t$ test. $\gamma_{1}$ and $\gamma_{2}$ are velocity constants obtained from the biexponential time function denoting the exchange of lactate and its removal during recovery, respectively (Engel et al. 2015).

boys and men could be useful in connection with more detailed investigations. However, neither model has been validated in boys with sophisticated invasive methods (which for us is problematic from an ethical point of view) and we believe on the basis of our argumentation here that under present circumstances the biexponential model is both acceptable and adequate for interpretation of data in this area.

\section{References}

Beneke, R., Hütler, M., Jung, M., and Leithäuser, R.M. 2005. Modeling the blood lactate kinetics at maximal short-term exercise conditions in children, adolescents, and adults. J. Appl. Physiol. 99: 499-504. doi:10.1152/japplphysiol. 00062.2005. PMID:16020438.

Dost, F.H. 1968. Grundlagen der Pharmakokinetik. Thieme, Stuttgart, Germany. pp. 29-62.

Dotan, R., and Falk, B. 2015. Discussion: The kinetics of blood lactate in boys during and following a single and repeated all-out sprints of cycling are different than in men - Child-adult differences in lactate kinetics. Appl. Physiol. Nutr. Metab. 40(6): This issue. doi:10.1139/apnm-2015-0057.

Dotan, R., Ohana, S., Bediz, C., and Falk, B. 2003. Blood lactate disappearance dynamics in boys and men following exercise of similar and dissimilar peaklactate concentrations. J. Pediatr. Endocrinol. Metab. 16: 419-429. PMID: 12705368.

Engel, F.A., Sperlich, B., Stockinger, C., Härtel, S., Bös, K., and Holmberg, H.C. 2015. The kinetics of blood lactate in boys during and following a single and repeated all-out sprints of cycling are different than in men. Appl. Physiol. Nutr. Metab. 40(6): This issue. doi:10.1139/apnm-2014-0370.

Freund, H., and Zouloumian, P. 1981. Lactate after exercise in man: I. Evolution kinetics in arterial blood. Eur. J. Appl. Physiol. Occup. Physiol. 46: 121-133. doi:10.1007/BF00428865. PMID:7194790.

Kappenstein, J., Engel, F., Fernandez-Fernandez, J., and Ferrauti, A. 2015. Effects of active and passive recovery on blood lactate and blood $\mathrm{pH}$ after a repeated sprint protocol in children and adults. Pediatr. Exerc. Sci. 27(1): 77-84. doi: 10.1123/pes.2013-0187. PMID:25387324.

Messonnier, L., Freund, H., Denis, C., Féasson, L., and Lacour, J. 2006. Effects of training on lactate kinetics parameters and their influence on short highintensity exercise performance. Int. J. Sports Med. 27: 60-66. doi:10.1055/s2005-837507. PMID:16388444.

Rimaud, D., Messonnier, L., Castells, J., Devillard, X., and Calmels, P. 2010. Effects of compression stockings during exercise and recovery on blood lactate kinetics. Eur. J. Appl. Physiol. 110: 425-433. doi:10.1007/s00421-010-1503-x. PMID: 20512586. 